\title{
BMJ Open Effectiveness of complementary food supplements and dietary counselling on anaemia and stunting in children aged 6-23 months in poor areas of Qinghai Province, China: a controlled interventional study
}

To cite: Zhang $\mathrm{Y}, \mathrm{Wu} \mathrm{Q}$, Wang W, et al. Effectiveness of complementary food supplements and dietary counselling on anaemia and stunting in children aged 6-23 months in poor areas of Qinghai Province, China: a controlled interventional study. BMJ Open 2016;6: e011234. doi:10.1136/ bmjopen-2016-011234

- Prepublication history for this paper is available online. To view these files please visit the journal online (http://dx.doi.org/10.1136/ bmjopen-2016-011234).

Received 21 January 2016 Revised 10 August 2016 Accepted 16 August 2016

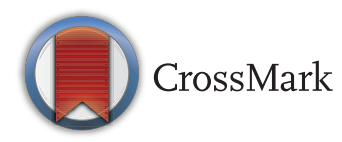

For numbered affiliations see end of article.

\section{Correspondence to}

Yanfeng Zhang, MSc, Department of Integrated Early Childhood

Development, Capital Institute of Pediatrics, No. 2 Yabao Road, Chaoyang District, Beijing 100020, China; summyzh@126.com. Suying Chang, PhD, Health and Nutrition, Water, Environment and Sanitation Section, UNICEF China, 12, Sanlitun Lu, Beijing 100600, China; schang@unicef.org

\begin{abstract}
Yanfeng Zhang, ${ }^{1}$ Qiong Wu, ${ }^{1}$ Wei Wang, ${ }^{1}$ Michelle Helena van Velthoven, ${ }^{2}$ Suying Chang, ${ }^{3}$ Huijun Han, ${ }^{4}$ Min Xing, ${ }^{5}$ Li Chen, ${ }^{1}$ Robert W. Scherpbier ${ }^{3}$
\end{abstract}

\section{ABSTRACT}

Objective: To assess the effectiveness of dietary counselling and complementary food supplements on anaemia and stunting prevalence in children aged 6-23 months.

Design: A controlled intervention study with measurements of height and haemoglobin levels, and cross-sectional surveys in August 2012 (baseline), 2013 (mid-term) and 2014 (end-line).

Setting: One intervention county and one control county in rural Qinghai Province, China.

Intervention: Complementary food supplements (containing protein, fat, carbohydrate, vitamin $A, B_{1}$, $B_{2}, B_{12}, D_{3}$, folic acid, iron, zinc and calcium) and complementary feeding counselling were given in the intervention county.

Participants: Caregivers and their children aged 6-23 months.

\section{Primary and secondary outcome measures:}

Effect of the interventions on the prevalence of anaemia (haemoglobin $<110 \mathrm{~g} / \mathrm{L}$ ) and stunting (z-score of height-for-age $<-2.0$ ) (controlled for differences between the counties), and on infant feeding practices.

Results: The surveys were conducted on 1804, 2187 and 2186 children aged 6-23 months in the intervention county in August 2012, 2013 and 2014, respectively, and 804,680 and 790 children in the control county, respectively. Between the baseline and end-line surveys, anaemia prevalence decreased more in the intervention county than in the control county ( $71.1 \%$ to $47.8 \%$ vs $86.3 \%$ to $75.3 \%$, respectively; $p<0.0001)$. There was no difference in the decrease in stunting prevalence between the counties $(9.7 \%$ to $7.1 \%$ vs $17.0 \%$ to $15.0 \% ; p=0.7954)$. The proportions of children given iron-rich or iron-fortified food, introduced to (semi-) solid food at 6-8 months, and given food with minimum dietary diversity increased from $43.2 \%$ to $88.8 \%$ ( $p<0.0001$ ), $81.4 \%$ to $96 \%$ $(p=0.0470)$ and $53.0 \%$ to $59.8 \%(p<0.0001)$, respectively in the intervention county.
Conclusions: We found much higher anaemia prevalence in poor rural areas of Qinghai Province compared with the national data. Community-based complementary food supplements combined with dietary counselling can improve feeding practices and reduce anaemia prevalence. Future studies should use longer follow-up to assess the effects on stunting. Strengths and limitations: We included a large number of participants and assessed a combined complementary food supplements and dietary counselling intervention in a poor rural area in China with high anaemia prevalence. Although the study took place in only one intervention county and one control county, we conducted an analysis that controlled for differences between the two counties. Also, although we made significant efforts to train village doctors, their education was not systematically assessed after training and thus their delivery of the interventions may have been variable.

Trial registration number: ChiCTRPRC12002444; Pre-results.

\section{BACKGROUND}

Malnutrition of children is still a large problem in China despite great achievements in improving children's nutrition over the past two decades. The estimated national prevalence of stunting among children under five in China was $9.9 \%$ in 2010, which is $70 \%$ lower than in $1990(33.4 \%)$ according to the 2012 National Report on the Nutritional Status of Children Aged 0-6 Years. ${ }^{1}$ The national prevalence of anaemia in children under five fluctuated between $12 \%$ and $23 \%$ from 1990 to 2005 , and then decreased from $19.3 \%$ in 2005 to $12.6 \%$ in 2010. ${ }^{1}$ However, in poor rural areas, the prevalence of stunting and anaemia among 
children under five was much higher than the national averages in 2010: the prevalence of stunting was $20.3 \%$, which was more than twice the national average $(9.9 \%)$, and the prevalence of anaemia in children aged 6-12 months and 13-24 months was $28.2 \%$ and $20.5 \%$, respectively. ${ }^{1}$

The key period for children's growth and development is $0-23$ months. It is recommended that children should be exclusively breast fed in the first 6 months after birth. However, breast milk alone is insufficient to meet all nutritional requirements after 6 months ${ }^{2}$ when infants enter a particularly vulnerable period of complementary feeding during which they have to adapt to eating family meals. In addition, there is a high demand for micronutrients from 6 to 23 months after birth to support infants' rapid growth and produce adequate development, which is hard to meet with plant-based diets. Therefore, the incidence of malnutrition rises sharply between 6 and 23 months after birth in most countries. ${ }^{3}$ Also, many children in rural China are not fed appropriately. Previous studies indicate that they usually eat food that contains mainly carbohydrates and lacks protein and fat. ${ }^{4}$ Animal source foods are rarely given to children as complementary foods, which is common in rural areas. ${ }^{5-7}$ These feeding problems result in a high prevalence of malnutrition in children under five in rural China. ${ }^{8}$ Therefore, more efforts are required to improve child feeding practices and thus improve the nutritional status of children.

Dietary diversification, food fortification, control of parasitic infections and nutritional supplements are the main interventions to reduce malnutrition in infants recommended by the WHO. ${ }^{9}$ Nutrition education programmes are recommended to improve complementary feeding by food diversity and proper use of local food. However, the high nutrition requirements of young children is far beyond the total intake of micronutrients in the small amount of food children consume. ${ }^{10}$ Therefore, home fortification of complementary foods with powders containing multiple micronutrients has been suggested in order to increase children's intake of vitamins and minerals; a mixture of micronutrients in powder form in single-serving sachets is added to the semisolid food that children consume. ${ }^{11}$ There is evidence that home fortification of foods with multiple micronutrient powders is an effective intervention to rapidly reduce anaemia and iron and vitamin A deficiency in young children. ${ }^{12}{ }^{13}$ WHO has developed guidelines on the use of multiple micronutrient powders for home fortification of foods for children aged 6-23 months ${ }^{14}$ based on a Cochrane Collaboration systematic review on the effects and safety of home fortification with multiple micronutrient powders. ${ }^{15}$

In China, a special complementary food supplement with multiple nutrients named 'Ying Yang Bao' (YYB) has been developed to prevent and reduce malnutrition in rural children. Compared with other multiple micronutrient powders in other countries, YYB contains essential fatty acids and protein through inclusion of full-fat soy flour as well as multiple micronutrients. In 2009, the Chinese government approved it and issued the National Standard for Complementary Food Supplements (GB/T22570-2008); ${ }^{16}$ YYB was then made commercially available. In October 2012, the National Health and Family Planning Commission and All-China Women's Federation jointly initiated a programme to improve children's nutrition in poor rural areas. The programme covered 100 counties in 10 provinces, and all children aged 6-23 months in the programme areas were provided with YYB free of charge. Qinghai Province, which was not covered by the national programme, used its own funds to provide YYB to all children aged 6-23 months in the 10 counties that have the highest anaemia and stunting prevalence.

Although studies in China showed that complementary food supplements were effective in reducing childhood anaemia, increasing weight and length and improving intellectual development, ${ }^{10}{ }^{17-20}$ those studies were either conducted on a small scale or had no control group to evaluate the effectiveness of complementary food supplements. Furthermore, the complementary food supplements used in those previous studies were different from YYB (the current nationally approved supplement). To our knowledge, no previous study has evaluated the effects of YYB in Qinghai Province. Therefore, this study aims to evaluate the effectiveness of YYB and health education in improving anaemia and stunting in children aged 6-23 months in Qinghai Province, and to provide policy recommendations to national and local government.

\section{MATERIALS AND METHODS \\ Study design}

This study was designed as a controlled intervention study and took place in two counties in Qinghai Province. In the intervention county, Huzhu County, children aged 6-23 months received the YYB complementary food supplement and their caregivers received infant feeding counselling, whereas in the control county, Guinan County, no interventions took place. Village doctors delivered the interventions.

\section{Study setting}

Qinghai Province lies in northwest China, with an area of $720000 \mathrm{~km}^{2}$. By the end of 2011, the total population was 5681700 , of which the rural population accounted for $53.8 \%$. Qinghai Province has 34 counties and 439 townships. The average rural net income was $¥ 4608$ per capita (US\$731.43) for rural people, which was similar to the national average ( $¥ 4761$ (US $\$ 755.71)$ ). ${ }^{21}$

This study was part of the Qinghai local YYB programme, in which the provincial government provided free complementary food supplements to children aged 6-23 months in poor counties. Before we designed the study, the provincial government had already decided to 
implement the programme in 15 counties. Therefore, we could only select an intervention county from one of these 15 counties. Most of the 15 YYB programme counties have large agricultural areas where mostly Han Chinese people live relatively close to each other. The remaining 19 counties, in which YYB was not introduced, were all Tibetan where the people are very scattered. When selecting the intervention and control counties, we took into consideration the willingness of the local government to cooperate and the socioeconomic conditions in the two counties, including the annual per capita income for rural residents, the adult female literacy rate, and the proportion of piped water coverage. However, inevitably there were differences between the intervention and control counties.

The selected intervention county, Huzhu County, lies in the northeast of Qinghai Province, with an area of $6650 \mathrm{~km}^{2}$. The total population was 370540 , of which the rural population accounted for $93.1 \%$ and more than $80 \%$ people were Han Chinese. People in Huzhu County mainly make a living by farming. Huzhu County has 19 townships and 294 villages. The annual per capita income of rural residents was $¥ 5691^{21}$ in 2011.

The selected control county, Guinan County, lies in the northeast of Qinghai Province, with an area of $3424 \mathrm{~km}^{2}$ and a total population of 76000 , of whom $75 \%$ are Tibetans. People in Guinan County mainly make a living by herding. Guinan County has 6 townships and 75 villages. The annual per capita income of rural residents was $¥ 4965^{21}$ in 2011.

The distance between the control county and the intervention county is $274 \mathrm{~km}$. Given this relatively large distance, families rarely move between the two counties and we do not expect contamination of the intervention county from the control county.

\section{Interventions and delivery channels}

The intervention was a daily complementary food supplement (YYB) for children aged 6-23 months. Caregivers of each child in the intervention county received one micronutrient powder sachet for each day of the month. Each sachet contained the following: protein $(3.0 \mathrm{~g})$, fat $(1.0 \mathrm{~g})$, carbohydrate $(3.0 \mathrm{~g})$, vitamin A $(250 \mu \mathrm{g})$, vitamin $\mathrm{D}_{3}(5 \mu \mathrm{g})$, vitamin $\mathrm{B}_{1}(0.5 \mathrm{mg})$, vitamin $B_{2}(0.5 \mathrm{mg})$, vitamin $\mathrm{B}_{12}(0.5 \mu \mathrm{g})$, folic acid $(75 \mathrm{mg})$, elemental iron $(7.5 \mathrm{mg})$, zinc $(5 \mathrm{mg})$ and calcium (200 mg) (table 1). In addition, village doctors provided reinforced infant feeding counselling to caregivers of children in the intervention county, which mainly focused on complementary feeding, including when to introduce complementary food, what could be given to children as complementary food, and the frequency and amount of complementary food that should be given to children at different ages (figure 1).

The complementary food supplements were distributed to caregivers of all the children aged 6-23 months in the intervention county during the study period from September 2012 to August 2014. The intervention was implemented through the rural three-tier healthcare system (county-township-village).

At county level, the Huzhu Maternal and Child Health (MCH) Hospital was responsible for programme management, including planning the programme, training and supervising the staff, and monitoring the outcomes.

At township level, we provided training to healthcare workers in township hospitals about feeding counselling and complementary food supplement-related information. Township healthcare workers were responsible for training and supervising staff and monitoring the programme in their catchment areas. Township healthcare workers trained village doctors during their regular

Table 1 Formulation of complementary food supplement in this study

\begin{tabular}{|c|c|c|c|c|c|}
\hline & \multirow{3}{*}{$\begin{array}{l}\text { Complementary food } \\
\text { supplement } \\
\text { Average level per } \\
\text { sachet }(12 \mathrm{~g})\end{array}$} & \multicolumn{4}{|c|}{ Chinese RNI or Al (2013) } \\
\hline & & \multicolumn{2}{|c|}{ 6-12 months } & \multicolumn{2}{|l|}{$1-3$ years } \\
\hline & & RNI or Al & $\%$ & RNI or Al & $\%$ \\
\hline Energy (kJ) & 200 & - & - & - & - \\
\hline Protein (g) & 3.0 & - & - & - & - \\
\hline Fat $(g)$ & 1.0 & - & - & - & - \\
\hline Carbohydrate (g) & 3.0 & - & - & - & - \\
\hline Vitamin A $(\mu \mathrm{g})$ & 250 & 350 & 71 & 310 & 81 \\
\hline Vitamin $D_{3}(\mu g)$ & 5 & 10 & 50 & 10 & 50 \\
\hline Vitamin $B_{1}(\mathrm{mg})$ & 0.5 & 0.3 & 167 & 0.6 & 83 \\
\hline Vitamin $B_{2}(m g)$ & 0.5 & 0.5 & 100 & 0.6 & 83 \\
\hline Vitamin $B_{12}(\mu \mathrm{g})$ & 0.5 & 0.6 & 83 & 1.0 & 50 \\
\hline Folic acid $(\mu \mathrm{g})$ & 75 & 100 & 75 & 160 & 47 \\
\hline Calcium (mg) & 200 & 250 & 80 & 600 & 33 \\
\hline Iron (mg) & 7.5 & 10 & 75 & 9 & 83 \\
\hline Zinc (mg) & 5 & 3.5 & 143 & 4.0 & 125 \\
\hline
\end{tabular}

$\mathrm{Al}$, adequate intake; $\mathrm{RNI}$, recommended nutrient intake. 


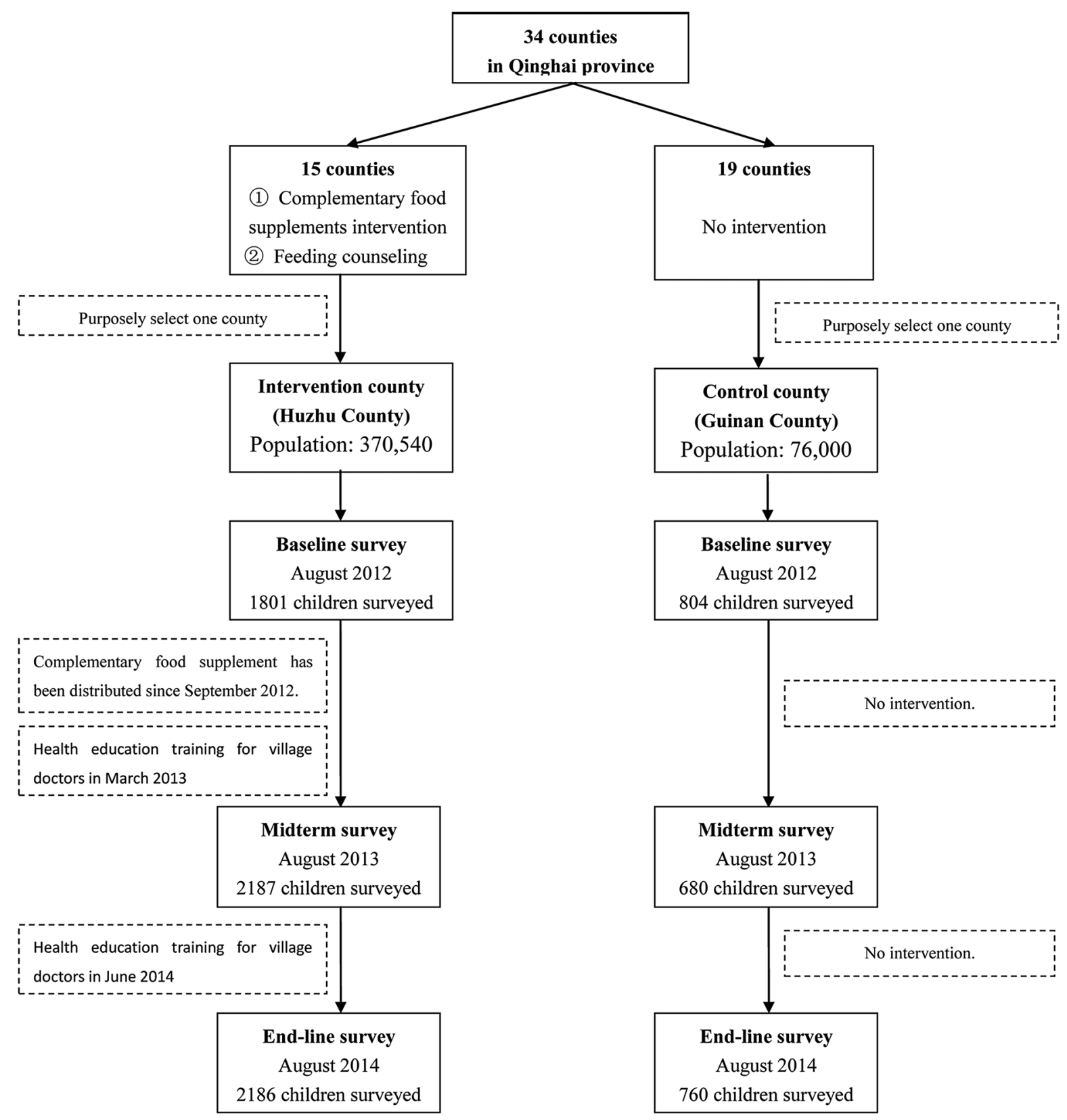

Figure 1 Flowchart of study procedures.

meeting in townships. Qinghai Health Education Center staff conducted two training courses for village doctors, the first in March 2013 and the second in June 2014.

Village doctors were responsible for distributing and advocating complementary food supplements, and providing feeding counselling to caregivers in their catchment areas. The box and complementary food supplement sachet contained key information on formulation of the micronutrient powder and its usage. Each caregiver also received one copy of health education material called 'A letter to caregivers', which contained instructions on how to give the complementary food supplement to children and the benefits of the supplement. When village doctors distributed complementary food supplements to caregivers, they explained the contents of the supplement box, gave health education material, delivered key infant feeding information, and answered caregivers' questions.

Children in the control county only received the usual child health service according to the national Basic Public Health Service (BPHS) programme in China, in which healthcare workers are required to provide breastfeeding counselling at antenatal visits, newborn home visits and postnatal visits, and complementary feeding counselling at child healthcare visits at the age of $1,3,6$, $8,12,18,24$ and 30 months. There was no complementary food supplement intervention or extra reinforced infant feeding counselling on the complementary feeding from the village in the control county during the study period. After the end-line survey (September 
2014), the complementary food supplement intervention was also implemented in the control county (Guinan) by the government.

\section{Participants}

Children and their caregivers in both counties were participants of our study. The inclusion criteria were as follows: (1) children aged between 6 and 23 months; (2) primary caregivers; and (3) rural children who could be distinguished by their urban or rural registration (known as Hukou in China), place of registration, and geographic location. The exclusion criteria were: (1) children with a structural or genetic birth defect, such as neural tube defects, congenital heart disease or phenylketonuria; (2) caregivers who refused to participate.

\section{Sample size and sampling}

We calculated the sample size required for this study on the basis of previously collected data. We expected to achieve a $20 \%$ reduction in the prevalence of stunting and anaemia. With a power of $80 \%$ and $5 \%$ significance level, we calculated that a sample size of 1793 children aged 6-23 months per group would be sufficient for all key indicators. We oversampled $30 \%$ of children to compensate for possible refusal and loss to follow-up.

We used a two-stage sampling procedure to select children for cross-sectional household surveys in the intervention county (Huzhu County). The two-stage sampling procedure was a standard sampling method for household-based surveys recommended in the guideline 'Indicators and methods for cross-sectional surveys of vitamin and mineral status of populations'. ${ }^{22}$ It recommended that communities, wards or villages were designated as the primary sampling units. First, the primary sampling units were required to be selected randomly, and then the households or subjects were selected randomly. In our survey, at the first stage, we selected 150 villages using proportional to population size sampling. At the second stage, we obtained a list of names of all eligible children aged 6-23 months in each village from the local maternal and child health hospital (all live births in the intervention county are reported to the maternal and child health hospital from all qualified delivery institutions every month), and we randomly selected 15 children per village using SAS V.9.2 software. We asked caregivers to first come to village clinics for registration, and then interviewers conducted interviews with caregivers there. We interviewed caregivers of 12 of 15 sampled children in each village based on the order in which they came to the village clinic (the first 12 children were selected).

Compared with the intervention county, the total population in the control county was smaller, with only about 1580 eligible children in the whole county. In addition, the control county was a Tibetan county, where people mainly make a living by herding. In summer, many Tibetans live as nomads away from their home, and take their children with them. Therefore, there were fewer children aged 6-23 months available at home, so we surveyed all the available children aged 623 months in the control county.

\section{Data collection}

We collected baseline data in August 2012 and distributed complementary food supplements for 2 years from September 2012. We conducted two follow-up surveys: the mid-term survey in August 2013 and the end-line survey in August 2014.

Staff from the Capital Institute of Pediatrics in Beijing acted as supervisors. We trained medical students from the School of Public Health, Qinghai University as interviewers to collect data in the study areas. We used the WHO Maternal, Newborn and Child Health (MNCH) Household Survey (unpublished, 2009) and the Demographic Health Survey (DHS) as the survey instruments to collect basic household information, socioeconomic indicators, breast feeding and nutrition, and children's intake of the complementary food supplements. We set up the household survey questionnaire in specially developed software on smartphones, ${ }^{23}$ which interviewers used to record data. Staff trained all surveyors for 2 days before the field work, including how to measure weight, height and haemoglobin of children using WHO standard measurement procedures.

In each survey, interviewers first introduced the aim of the survey to caregivers and obtained written informed consent from them. Then the interviewers asked questions following instructions on the smartphone. We saved data of each questionnaire on the memory card of the smartphone as a text file. Once the interview was completed, we measured the length and haemoglobin level of each child following WHO standard procedures. The surveyors measured the recumbent length of the children with a locally produced infant-length-measuring board accurate to the nearest $0.1 \mathrm{~cm}$. We performed each length measurement twice. If there was more than $0.5 \mathrm{~cm}$ difference between two measurements, an additional measurement was undertaken until the difference was $<0.5 \mathrm{~cm}$. The average of the two measurements was used as the final value. Another surveyor measured haemoglobin with a HemoCue Hb301 analyser (HemoCue, Lake Forest, CA, USA) by drawing about $10 \mu \mathrm{L}$ blood from the finger. Two people separately entered length and haemoglobin data with EpiData 3.1. We compared the two files, and discrepancies were resolved by referring to the original records.

\section{Outcome}

\section{Primary outcome}

Our primary outcomes were anaemia and stunting prevalence. As both the intervention county and the control county are more than $2000 \mathrm{~m}$ above sea level, we adjusted all individual haemoglobin values based on the altitude using WHO recommendations. ${ }^{24}$ We used the altitude in each village surveyed to adjust the 
haemoglobin and then compared the adjusted haemoglobin values with the sea level anaemia status cut-off point (haemoglobin $<110 \mathrm{~g} / \mathrm{L}$ ) to calculate the anaemia prevalence of both counties in the three surveys. A haemoglobin concentration of $90-110 \mathrm{~g} / \mathrm{L}$ was defined as mild anaemia, $70-90 \mathrm{~g} / \mathrm{L}$ as moderate anaemia, and $<70 \mathrm{~g} / \mathrm{L}$ as severe anaemia. We analysed length-for-age with the WHO Anthro software. ${ }^{25}$ We defined stunting as having a z-score $<-2.0$ for length-for-age.

\section{Secondary outcome}

Secondary outcomes were five core feeding practice indicators, which were calculated according to the WHO guidelines 'Indicators for assessing infant and young child feeding practices ${ }^{26}$ (box 1), and adherence to the complementary food supplement.

\section{Data security}

All data were encrypted and stored in the smartphones when the data were uploaded. The supervisors collected the smartphones at the end of each field working day and returned them (cleared of data entered the previous day) to the interviewers in the morning. We manually transformed and pooled the data from the questionnaires into a Microsoft Excel sheet. After data cleaning, we converted the database into a database file (dbf) for final analysis.

\section{Data analysis}

We carried out statistical analysis with SAS V.9.2 for Windows. We summarised sociodemographic, feeding practice, complementary food supplement consumption and nutrition characteristics of children as medians and

\section{Box 1 WHO core feeding practice indicators}

- Introduction of solid, semisolid or soft foods: proportion of infants 6-8 months of age who receive solid, semisolid or soft foods.

- Minimum dietary diversity. the indicator was four out of seven food groups per day. The proportion of children aged 6-23 months who receive foods from four or more food groups was estimated. The food groups were: (1) grains, root and tubers; (2) legumes and nuts; (3) dairy products (milk, yogurt and cheese); (4) meat (meat, fish, poultry and liver/organ meat); (5) eggs; (6) vitamin A-rich fruits and vegetables; (7) other fruits and vegetables.

- Minimum meal frequency. proportion of breastfed and nonbreastfed children aged 6-23 months who received solid, semisolid or soft foods (also including milk for non-breastfed children) the minimum number of times or more.

- Minimum acceptable diet. proportion of children aged 6-23 months who reached a minimum dietary diversity and minimum meal frequency.

- Consumption of iron-rich or iron-fortified foods: proportion of children aged 6-23 months who received iron-rich or ironfortified food that was specially designed for infants and young children or that was fortified in the home. quartiles $(\mathrm{Q} 1, \mathrm{Q} 3)$ for continuous variables and as frequencies for categorical variables.

We estimated high adherence as children who took at least $60 \%$ of the recommended amount of YYB, which was calculated by dividing the total number of boxes of YYB consumed by the children up until the time of our surveys by the number of boxes children should take from 6 months until the time of the surveys.

As the age distribution of surveyed children differed between the intervention and control counties in the three surveys, we adjusted the feeding practice indicators and the prevalence of anaemia and stunting by using the total number of children in the three surveys as the standard population.

We used a difference-in-difference (D-in-D) estimation to assess the effects of the intervention on anaemia, stunting and other infant and young child feeding indicators in a linear model. The D-in-D estimation is the coefficient of the interaction between the intervention and time in a regression model, with intervention, time and their interaction as covariates. As we selected the intervention and control counties arbitrarily, this method corrects for differences between the intervention and control groups, as everything apart from the variable of interest (or other aspects that can be controlled for) is assumed to be the same. As for anaemia, stunting and other infant and young child feeding indicators, the outcome was the prevalence, within the range $0-1$. Therefore, the $95 \%$ CI was then calculated with bootstrapping, with a replication of 2000 .

\section{Ethics considerations}

The ethics committee of the Capital Institute of Pediatrics in Beijing approved the study (reference number 2012057). All interviewees read the information sheet and provided oral and written consent on behalf of the children involved in the study.

\section{RESULTS}

The baseline, mid-term and end-line surveys were conducted on 1804, 2187 and 2186 children aged 6-23 months in the intervention county in August 2012, 2013 and 2014, respectively, and 804, 680 and 790 children in the control county, respectively. Table 2 shows that, at baseline, more mothers worked outside the county in the intervention county $(24.2 \%)$ than in the control county $(6.7 \%)$. In both counties, nearly all main caregivers were mothers and grandparents, with a higher percentage of mothers in the control county than in the intervention county. The proportion of main caregivers who never attended primary school and were illiterate was lower in the intervention county $(41.3 \%)$ than in the control county $(61.4 \%)$. Feeding practices were generally suboptimal in both counties, but they were worse in the intervention county. However, anaemia and stunting prevalence were both higher in the control than in the intervention county. Anaemia and stunting 
Table 2 Baseline characteristics of the intervention and control groups

\begin{tabular}{|c|c|c|c|c|c|}
\hline Characteristic & $\begin{array}{l}\text { Intervention group } \\
(\mathrm{N}=1804)\end{array}$ & $\begin{array}{l}\text { Control } \\
\text { group } \\
(\mathrm{N}=804)\end{array}$ & $\begin{array}{l}\text { Adjusted } \\
\text { intervention group }\end{array}$ & $\begin{array}{l}\text { Adjusted } \\
\text { control group }\end{array}$ & p Value \\
\hline \multicolumn{6}{|l|}{ Household } \\
\hline Size (median (Q1,Q3)) & $5(5,6)$ & $5(4,6)$ & - & - & 0.0034 \\
\hline Safe drinking water supply (\%) & 91.4 & 75.0 & - & - & $<0.0001$ \\
\hline Sanitary toilet (\%) & 7.8 & 10.2 & - & - & 0.0425 \\
\hline $\begin{array}{l}\text { Water at the place for hand } \\
\text { washing (\%) }\end{array}$ & 70.2 & 61.3 & - & - & $<0.0001$ \\
\hline $\begin{array}{l}\text { Soap, detergent or other cleaning } \\
\text { agent }(\%)\end{array}$ & 90.9 & 78.4 & - & - & $<0.0001$ \\
\hline \multicolumn{6}{|l|}{ Mothers } \\
\hline Age in years (median (Q1, Q3)) & $26(23,30)$ & $25(23,29)$ & - & - & 0.0035 \\
\hline Working outside county (\%) & 24.2 & 6.7 & - & - & $<0.0001$ \\
\hline \multicolumn{6}{|l|}{ Main caregivers } \\
\hline \multicolumn{6}{|l|}{ Relationship to children } \\
\hline Mother (\%) & 53.2 & 68.3 & - & - & $<0.0001$ \\
\hline Father (\%) & 0.6 & 0.9 & - & - & \\
\hline Grandparent (\%) & 45.0 & 30.2 & - & - & \\
\hline Other (\%) & 1.2 & 0.6 & - & - & \\
\hline Age in years (median (Q1, Q3)) & $50(36,58)$ & $29(24,45)$ & & & $<0.0001$ \\
\hline Illiterate (\%) & 41.3 & 61.4 & - & - & $<0.0001$ \\
\hline \multicolumn{6}{|l|}{ Children } \\
\hline Age in months (median (Q1, Q3)) & $15(10,20)$ & $14(9,18)$ & - & - & $<0.0001$ \\
\hline Age $6-11$ months $(\%)$ & 33.8 & 38.9 & - & - & $<0.0001$ \\
\hline Age $12-17$ months (\%) & 26.8 & 31.3 & - & - & \\
\hline Age $18-23$ months (\%) & 39.4 & 29.7 & - & - & \\
\hline \multicolumn{6}{|l|}{ Sex } \\
\hline Male (\%) & 53.2 & 52.2 & - & - & 0.6446 \\
\hline Female (\%) & 46.8 & 47.8 & - & - & \\
\hline \multicolumn{6}{|c|}{ Feeding practice, anaemia and stunting } \\
\hline $\begin{array}{l}\text { Introduction of solid, semisolid or } \\
\text { soft foods at } 6-8 \text { months }(\%)^{*}\end{array}$ & 81.2 & 77.9 & 81.4 & 79.2 & 0.3941 \\
\hline $\begin{array}{l}\text { Reaching minimum dietary } \\
\text { diversity }(\%)\end{array}$ & 51.5 & 32.6 & 53.0 & 34.6 & $<0.0001$ \\
\hline $\begin{array}{l}\text { Reaching minimum meal } \\
\text { frequency }(\%)\end{array}$ & 28.2 & 33.5 & 26.7 & 31.7 & 0.0063 \\
\hline $\begin{array}{l}\text { Reaching minimum acceptable } \\
\text { diet }(\%)\end{array}$ & 5.5 & 7.3 & 10.0 & 8.2 & 0.0769 \\
\hline $\begin{array}{l}\text { Children aged } 6-23 \text { months given } \\
\text { iron-rich or iron-fortified foods (\%) }\end{array}$ & 41.5 & 47.5 & 43.2 & 51.4 & 0.0043 \\
\hline Anaemic (\%) & 71.1 & 86.3 & 70.4 & 84.8 & $<0.0001$ \\
\hline Stunted (\%) & 9.7 & 17.0 & 9.9 & 17.8 & $<0.0001$ \\
\hline
\end{tabular}

prevalence was very high in both countries: $70.4 \%$ and $9.9 \%$ in the intervention and $84.8 \%$ and $17.8 \%$ in the control county.

Figure 2 shows that, in the intervention county, three indicators increased significantly between the baseline and end-line surveys: the proportions of children who were given iron-rich or iron-fortified foods, children who were introduced to solid or semisolid food, and children who reached the minimum dietary diversity target increased from $43.2 \%$ to $88.8 \%$ ( $p<0.0001), 81.4 \%$ to $96 \% \quad(p=0.0470)$ and $53.0 \%$ to $59.8 \% \quad(p<0.0001)$, respectively. However, in the control county, these three indicators either decreased or increased slightly. There was almost no change in minimum meal frequency and minimum acceptable diet in the intervention county, whereas in the control county minimum meal frequency slightly increased and minimum acceptable diet decreased.

For children's intake of the complementary food supplement, the end-line survey showed that nearly all surveyed children $(98.1 \%)$ at sometime took the complementary food supplement, and $62.4 \%$ of them had $60.0 \%$ or more of the recommended amount (table 3). The complementary food supplements were consumed by $78.8 \%$ of the 

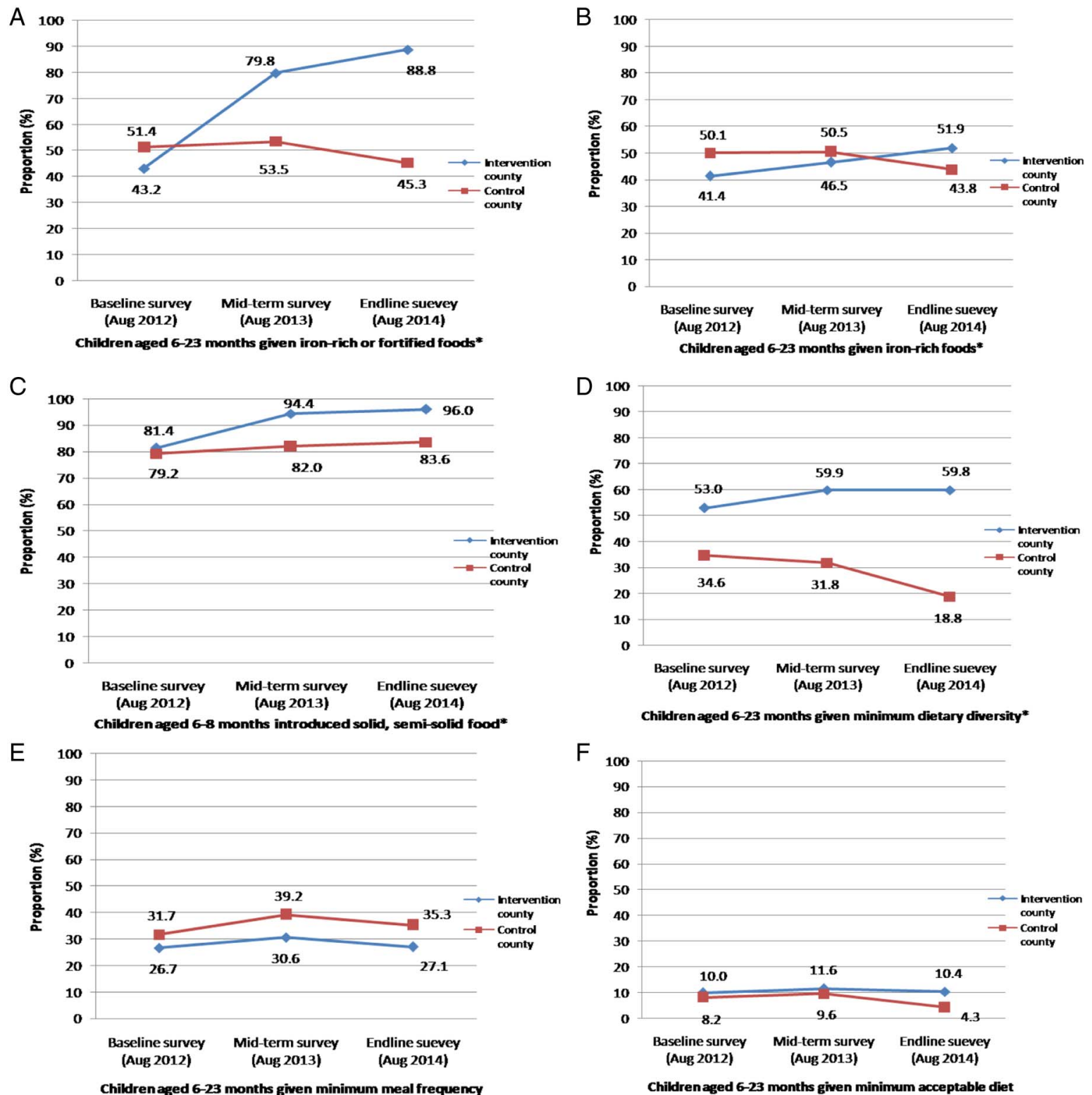

Figure 2 Adjusted feeding practices of children in the three surveys. (A) Children given iron-rich or iron-fortified food: intervention baseline vs end-line, $p<0.0001$; net effect intervention vs control, $p<0.0001$. (B) Children given iron-rich food: intervention baseline vs end-line survey, $p<0.0001$; net effect of intervention vs control, $p<0.0001$. (C) Introduction of solid or semisolid food: intervention baseline vs end-line survey, $p<0.0001$; net effect of intervention vs control, $p=0.0470$. (D) Minimum diet diversity: intervention baseline vs end-line survey, $p<0.0001$; net effect of intervention vs control, $p<0.0001$. (E) Minimum meal frequency: intervention baseline vs end-line survey, $p=0.5219$; net effect of intervention vs control, $p=0.0702$. (F) Minimum acceptable diet: intervention baseline vs end-line survey, $p=0.5396$; net effect of intervention vs control, $p=0.0057$. ${ }^{*}$ The $p$ values were significant in these figures.

children within the last 24 hours, and $87.0 \%$ consumed 5-7 sachets during the last week in the end-line survey.

Table 4 shows that, in the intervention county, the prevalence of total anaemia, mild anaemia and moderate and severe anaemia continued to decrease from the baseline to the mid-term and end-line survey. In the control county, the prevalence of total anaemia and moderate and severe anaemia also continued to decrease in all three surveys, but the prevalence of mild anaemia increased in the mid-term and end-line survey compared with the baseline. There were significant net effects for total anaemia and mild anaemia in both the mid-term and end-line surveys between the counties, but the net effects for moderate and severe anaemia were not significant $(\mathrm{p}=0.7687$ and $\mathrm{p}=0.8475$, respectively). Further analysis of YYB consumption in the end-line survey shows that children who were non-anaemic or mildly anaemic took a median of $7(6,7)$ sachets of YYB, and children with moderate or severe anaemia took 7 $(4,7)$ sachets of YYB; children who were non-anaemic or 
Table 3 Children's intake of complementary food supplements

\begin{tabular}{|c|c|c|c|}
\hline Indicator & $\begin{array}{l}\text { Baseline } \\
\text { survey } \\
(\mathrm{N}=1804)\end{array}$ & $\begin{array}{l}\text { Mid-term } \\
\text { survey } \\
\left(\mathrm{N}=2186^{\star}\right)\end{array}$ & $\begin{array}{l}\text { End-line } \\
\text { survey } \\
(\mathrm{N}=2186)\end{array}$ \\
\hline Ever consumed the complementary food supplement & - & $95.9(2096)$ & $98.1(2144)$ \\
\hline Consumed the complementary food supplement within the last 24 hours & - & $63.3(2144)$ & $78.8(1722)$ \\
\hline $\begin{array}{l}\text { High adherence (took } \geq 60 \% \text { of the recommended amount of } \\
\text { complementary food supplement) }\end{array}$ & - & $37.0(573)$ & $62.4(936)$ \\
\hline \multicolumn{4}{|l|}{$\begin{array}{l}\text { Sachets of complementary food supplement that } \\
\text { children consumed during the last week }\end{array}$} \\
\hline $5-7$ & - & 76.1 & 87.0 \\
\hline $3-4$ & - & 14.1 & 7.9 \\
\hline $1-2$ & - & 6.2 & 3.3 \\
\hline 0 & - & 3.6 & 1.8 \\
\hline
\end{tabular}

mildly anaemic took more YYB than children who were moderately or severely anaemic in the end-line survey $(\mathrm{p}=0.001)$.

Table 5 shows that the adjusted stunting prevalence continued to decrease from the baseline to the mid-term and end-line surveys in both counties. The difference in the net effect between the two counties was not significant for stunting $(\mathrm{p}=0.8313$ and $\mathrm{p}=0.7954$, respectively).

\section{DISCUSSION}

This controlled interventional study assessed the effectiveness of a daily complementary food supplement and dietary counselling for reducing the prevalence of anaemia and stunting in children aged 6-23 months in rural areas of Qinghai Province, China. The intervention assessed was the first large-scale community-based nutrition intervention aiming to improve dietary practices and decrease anaemia and stunting prevalence of children in poor areas in rural China. Our study indicated that, after 2 years of implementation of the interventions, three core feeding practices were improved (children aged 6-23 months given iron-rich or iron-fortified food, introduction of solid or semisolid food at 6-8 months, minimum dietary diversity), whereas the other two did not change (minimum meal frequency and minimum acceptable diet). The prevalence of total anaemia, mild anaemia, and moderate and severe anaemia continuously decreased in the intervention group. The difference in the net effect between the two counties was significant for total anaemia and mild anaemia prevalence, but not significant for moderate and severe anaemia. The prevalence of stunting continued to decrease from the baseline to mid-term and end-line surveys in both counties. However, the difference in the net effect between the two counties was not significant for stunting.

Although national data showed that the prevalence of anaemia for children under five in rural areas in China decreased to $13.3 \%$ in $2010,{ }^{1}$ the baseline data in our study showed that the prevalence of anaemia was extremely high: more than $70 \%$ in both counties. As a prevalence of anaemia of $5-12 \%$ is considered to be a low-grade public health problem and 12-19\% a medium-grade problem, ${ }^{27}$ it was urgent to take action to improve the nutritional status of children in these counties. During this study, the prevalence of anaemia in the intervention group decreased from $71.1 \%$ at the baseline to $57.1 \%$ at the 1-year follow-up, and continued to decrease to $47.8 \%$ at the 2-year follow-up. The interventions were effective in reducing anaemia prevalence in children aged 6-23 months by $12.7 \%$ compared with the control group (net effect). Although anaemia reduction was also observed in the control county, which can be attributed to natural reduction, it was smaller than the reduction in the intervention county. This result is similar to the results of previous studies in both China ${ }^{10}$ and several other developing counties, ${ }^{12} 1528$ which suggests that a daily micronutrient supplement is an effective treatment for reducing anaemia in young children.

Although our study had achieved a significant reduction in anaemia after 2 years of the intervention, still $\sim 48 \%$ of children aged 6-23 months were anaemic, which was much higher than the national level $(20-30 \%) .^{1}$ There are three possible explanations for this relatively low reduction in anaemia prevalence in the intervention group. First, although the amount of elemental iron in each sachet of the complementary food supplement was $7.5 \mathrm{mg}$, accounting for $75 \%$ of the recommended nutrient intake (RNI) stated by the Chinese Nutrition Society, it may not be sufficient to produce a haemoglobin response that can prevent and reduce anaemia in children in the study area. A Cochrane Review of eight trials on home fortification of foods with multiple micronutrient powder for health and nutrition in children under 2 years of age showed that a dose of $12.5 \mathrm{mg}$ iron along with $5 \mathrm{mg}$ zinc and $300 \mu \mathrm{g}$ vitamin $\mathrm{A}$ is effective for children $<2$ years old. ${ }^{15}$ According to the Chinese standard for fortified 


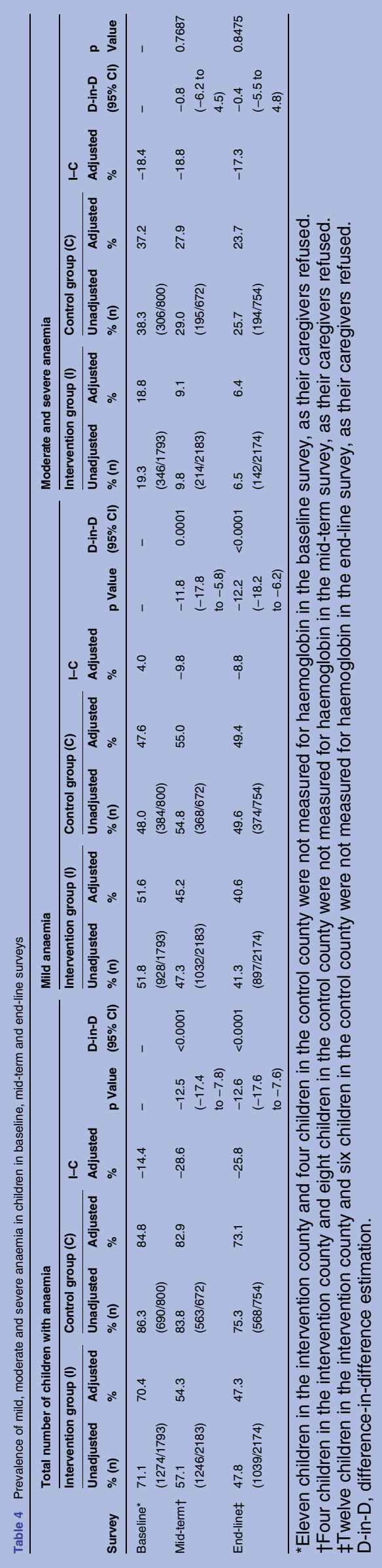

complementary food supplements (GB/T22570-2008), daily rations for iron in the complementary food supplement were not more than $90 \%$ of the RNI, and the Chinese Ministry of Health approved the addition of $7.5 \mathrm{mg}$ iron to the complementary food supplement. The Chinese government may need to reconsider the iron dose in the complementary food supplement. Second, the adherence of caregivers to giving the complementary food supplement to their child was suboptimal in our study. It is well known that adherence and acceptability of a product are essential for an intervention to be implemented successfully. The end-line data indicate that nearly one-third of the children did not take $60 \%$ or more of the recommended amount of the complementary food supplement. Third, there may have been other types of anaemia in the study areas apart from the iron-deficient form.

Nutrition education in food-secure populations significantly increased height and height-for-age z-scores. ${ }^{13}$ Also increasing dietary diversity was positively associated with height-for-age z-score. ${ }^{29}$ However, no studies have so far shown that the micronutrient supplement had an effect on growth. ${ }^{14}$ Although we combined daily complementary food supplements with nutrition education, we did not observe a difference in the stunting prevalence after a 2 year intervention. Our results show that, at the end-line survey, three key feeding practices were improved (children aged 6-23 months given iron-rich or ironfortified food, introduction of solid or semisolid food at 6-8 months, minimum dietary diversity), whereas the other two did not change (minimum meal frequency and minimum acceptable diet). These findings indicate that training village doctors to deliver health education could be effective in improving caregivers' feeding practices. However, $\sim 40 \%$ of the children in our study still did not achieve sufficient food diversity; only $\sim 10 \%$ of the children reached a minimum acceptable diet, which may be one of the reasons why the prevalence of stunting did not change. Also, the lack of effect of our interventions on children's height may have been because the carbohydrate and protein content of the complementary foods consumed by the children was too low. Despite the addition of $4.0 \mathrm{~g}$ protein and fat from soybeans to the complementary food supplement in each sachet, this may have been insufficient to benefit the children's growth. Moreover, a longer follow-up may be required to find a change in stunting prevalence. Another possible reason is poor sanitation in study areas. Environmental enteropathy (due to frequent intestinal infections), inflammation and other physiological responses to environmental factors (including mycotoxins and household air pollution) are likely to be far more common than clinically obvious infections and thus may account for a large amount of stunting in young children. ${ }^{30-34}$

\section{CONCLUSION}

We found a much higher prevalence of anaemia in poor rural areas of Qinghai Province compared with data 
Table 5 Prevalence of stunting in children in baseline, mid-term and end-line surveys

\begin{tabular}{|c|c|c|c|c|c|c|c|}
\hline \multirow[b]{2}{*}{ Survey } & \multicolumn{2}{|c|}{ Intervention group (I) } & \multicolumn{2}{|l|}{ Control group (C) } & \multirow{2}{*}{$\begin{array}{l}\text { I-C } \\
\text { Adjusted \% }\end{array}$} & \multirow[b]{2}{*}{ D-in-D (95\% Cl) } & \multirow[b]{2}{*}{ p Value } \\
\hline & Unadjusted \% (n) & Adjusted \% & Unadjusted \% (n) & Adjusted \% & & & \\
\hline Baseline* & $9.7(171 / 1770)$ & 9.9 & $17.0(133 / 783)$ & 17.8 & -7.9 & - & - \\
\hline Mid-term† & $9.0(194 / 2162)$ & 9.7 & $17.1(114 / 667)$ & 17.2 & -7.5 & $-0.4(-4.7$ to 3.9$)$ & 0.8313 \\
\hline End-line $\ddagger$ & $7.1(152 / 2150)$ & 7.2 & $15.0(112 / 746)$ & 14.9 & -7.7 & $-0.6(-4.8$ to 3.5$)$ & 0.7954 \\
\hline
\end{tabular}

*Thirty-four children in the intervention county and 21 children in the control county were not included in the baseline survey, because of abnormal or missing data.

†Twenty-five children in the intervention county and 13 children in the control county were not included in the mid-term survey, because of abnormal or missing data.

¥Thirty-six children in the intervention county and 14 children in the control county were not included in the end-line survey, because of abnormal or missing data.

D-in-D, difference-in-difference estimation.

from national surveys. Complementary food supplements and infant feeding counselling delivered by village doctors were effective in improving caregivers' feeding practices and reducing child anaemia. Longer follow-ups to assess the effects on stunting and implementation of other effective interventions are needed to improve the nutritional status of rural Chinese children.

\section{Strengths and limitations}

The strength of our study is that we included a large number of participants and combined an intervention of dietary counselling and complementary food supplement (YYB). Both dietary improvement and micronutrient supplement are important for improving child nutrition. However, our study also has some limitations. First, we were limited in our choice of intervention and control counties because the provincial government selected the counties in which complementary food supplements were provided. Although there were differences between the intervention and control counties, we conducted an analysis that controlled for these differences. As the decrease in anaemia prevalence was greater in the intervention county than in the control county, it is likely that the intervention had a positive effect on the measured indicators. Second, the education of village doctors was not systematically assessed after training and thus may have been variable. However, we made every attempt to train village doctors properly so that they were able to provide appropriate health education to the caregivers.

\section{Author affiliations}

${ }^{1}$ Department of Integrated Early Childhood Development, Capital Institute of Pediatrics, Beijing, China

${ }^{2}$ Health and Nutrition, Water, Environment and Sanitation Section, UNICEF China, Beijing, China

${ }^{3}$ Global eHealth Unit, Department of Primary Care and Public Health, Imperial College London, London, UK

${ }^{4}$ Department of Epidemiology and Biostatistics, Institute of Basic Medical Sciences Chinese Academy of Medical Sciences, School of Basic Medicine Peking Union Medical College, Beijing, China

${ }^{5}$ Department of Health Education in Framing and Pastoral Areas, Qinghai Health Education Center, Qinghai, China

Acknowledgements We wish to thank all colleagues from the Huzhu Maternal and Child Health Hospital and Guinan Health \& Family Planning Commission in Qinghai for coordination and logistic arrangements, and we want to thank all students from Qinghai University for their hard work as interviewers. We are indebted to all the mothers and caregivers who participated in our survey.

Contributors RWS, SC, YZ, QW, WW and HH designed the study; QW, WW and MX collected and analysed data; RWS, SC, YZ, QW, MHvV and LC interpreted data; $\mathrm{YZ}$ and MHvV wrote the first and subsequent drafts of the paper; all authors reviewed the paper and approved the publication.

Funding The study was funded by the United Nations Children's Fund (UNICEF). The funder was involved in study design, data interpretation, preparation of the manuscript, and decision to publish.

Competing interests None declared.

Data sharing statement Additional data can be accessed via the Dryad data repository at http://datadryad.org/ with the doi:10.5061/dryad.52mt5

Open Access This is an Open Access article distributed in accordance with the Creative Commons Attribution Non Commercial (CC BY-NC 4.0) license, which permits others to distribute, remix, adapt, build upon this work noncommercially, and license their derivative works on different terms, provided the original work is properly cited and the use is non-commercial. See: http:// creativecommons.org/licenses/by-nc/4.0/

\section{REFERENCES}

1. Ministry of Health China. National report on nutritional status of children aged 0-6 year (2012). Beijing, 2012.

2. Michael S, Kramer RK. The optimal duration of exclusive breastfeeding: a systematic review. World Health Organization, 2001.

3. PAHO. Guiding principles for complementary feeding of the breastfed child. 2003.

4. Chunming C. Fat intake and nutritional status of children in China. Am J Clin Nutr 2000;72:1368S-72S.

5. Yi C, Fang G, Zhang F. Survey of status of complementary food in Li ethnic infants in rural areas of Hainan Province. Zhong Guo Re Dai Yi Xue 2007;7:638-9.

6. Liu A, Zhao L, Yu D, et al. [Study on feeding status of infants and young children under 2-years-old in China]. Wei Sheng Yan Jiu 2009;38:555-7. (in Chinese)

7. Ye JL, Cui Y, Pan XP. [Cross-sectional study on the current situation of breast feeding in western China rural areas]. Zhonghua Yu Fang Yi Xue Za Zhi 2007;41:183-5. (in Chinese)

8. Chang $\mathrm{S}, \mathrm{He} \mathrm{W}, \mathrm{Chen} \mathrm{CM}$. [The growth characteristics of children under 5 in the past 15 years]. Wei Sheng Yan Jiu 2006;35:768-71. (in Chinese)

9. DeMaeyer EM. Preventing and controlling iron deficiency anaemia through primary health care: a guide for health administrators and programme managers. World Health Organization, 1989.

10. Wang YY, Chen CM, Wang FZ, et al. Effects of nutrient fortified complementary food supplements on anemia of infants and young children in poor rural of Gansu. Biomed Environ Sci 2009;22:194-200.

11. Zlotkin $\mathrm{SH}$, Schauer C, Christofides A, et al. Micronutrient sprinkles to control childhood anaemia. PLoS Med 2005;2:e1.

12. Suchdev PS, Ruth LJ, Woodruff BA, et al. Selling Sprinkles micronutrient powder reduces anemia, iron deficiency, and vitamin A deficiency in young children in Western Kenya: a cluster-randomized controlled trial. Am J Clin Nutr 2012;95:1223-30. 
13. Bhutta ZA, Das JK, Rizvi A, et al. Evidence-based interventions for improvement of maternal and child nutrition: what can be done and at what cost? Lancet 2013;382:452-77.

14. World Health Organization. Use of multiple micronutrient powders for home fortification of foods consumed by infants and children 6-23 months of age. World Health Organization, 2011.

15. De-Regil LM, Suchdev PS, Vist GE, et al. Home fortification of foods with multiple micronutrient powders for health and nutrition in children under two years of age (Review). Evid Based Child Health 2013;8:112-201.

16. Ministry of Health China, Standardization Administration of the People's Republic of China. Chinese standard for fortified complementary food supplements. Ministry of Health of the People's Republic of China, 2008 .

17. Wang $Y Y$, Wang FZ, Wang $\mathrm{K}$, et al. [Effects of nutrient fortified complementary food supplements on development of infants and young children in poor rural area of Gansu Province]. Wei Sheng Yan Jiu 2006;35:772-4. (in Chinese)

18. Wang $\mathrm{Y}$, Chen $\mathrm{C}$, Wang $\mathrm{F}$, et al. [Effects of nutrient fortified complementary food supplements on growth of infants and young children in poor rural area in Gansu Province]. Wei Sheng Yan Jiu 2007;36:78-81. (in Chinese)

19. Chen CM, Wang YY, Chang SY. Effect of in-home fortification of complementary feeding on intellectual development of Chinese children. Biomed Environ Sci 2010;23:83-91.

20. Sun J, Dai Y, Zhang S, et al. Implementation of a programme to market a complementary food supplement (Ying Yang Bao) and impacts on anaemia and feeding practices in Shanxi, China. Matern Child Nutr 2011;7 Suppl 3:96-111.

21. Qinghai Bureau of Statistics. Qinghai Statistics Year Book 2010. Beijing: China Statistics Press, 2011.

22. Micronutrient Initiative, Centers for Disease Control and Prevention (Atlanta). Indicators and methods for cross-sectional surveys of vitamin and mineral status of populations. May, 2007.
23. Zhang $\mathrm{S}, \mathrm{Wu} \mathrm{Q}$, van Velthoven $\mathrm{MH}$, et al. Smartphone versus pen-and-paper data collection of infant feeding practices in rural China. J Med Internet Res 2012;14:e119.

24. World Health Organization. Haemoglobin concentrations for the diagnosis of anaemia and assessment of severity. Geneva: World Health Organization, 2011.

25. WHO. WHO Anthro (version 3.2.2, January 2011) and macros. Geneva, 2011.

26. WHO, UNICEF, USAID, AED, UCDAVIS, IFPRI. Indicators for assessing infant and young child feeding practices-part 1 Introduction. WHO, 2010.

27. F V. Prevention of micronutrient deficiencies: tools for policymakers and public health workers. Washington, DC: Health Workers National Academy Press, 1998

28. Kounnavong S, Sunahara T, Mascie-Taylor CG, et al. Effect of daily versus weekly home fortification with multiple micronutrient powder on haemoglobin concentration of young children in a rural area, Lao People's Democratic Republic: a randomised trial. Nutr J 2011;10:129.

29. Arimond M, Ruel MT. Dietary diversity is associated with child nutritional status: evidence from 11 demographic and health surveys. J Nutr 2004;134:2579-85.

30. Dewey KG, Mayers DR. Early child growth: how do nutrition and infection interact? Matern Child Nutr 2011;7 Suppl 3:129-42.

31. Khlangwiset $P$, Shephard GS, Wu F. Aflatoxins and growth impairment: a review. Crit Rev Toxicol 2011;41:740-55.

32. Smith KR, McCracken JP, Weber MW, et al. Effect of reduction in household air pollution on childhood pneumonia in Guatemala (RESPIRE): a randomised controlled trial. Lancet 2011;378:1717-26.

33. Smith LE, Stoltzfus RJ, Prendergast A. Food chain mycotoxin exposure, gut health, and impaired growth: a conceptual framework. Adv Nutr 2012;3:526-31.

34. Prendergast A, Kelly P. Enteropathies in the developing world: neglected effects on global health. Am J Trop Med Hyg 2012;86:756-63. 